\title{
Protée
}

\section{Répercussions violentes : Échos de l’oeuvre de Thomas Bernhard}

\section{Simon Harel}

Volume 35, numéro 1, printemps 2007

Échos et résonances

URI : https://id.erudit.org/iderudit/015885ar

DOI : https://doi.org/10.7202/015885ar

Aller au sommaire du numéro

\section{Éditeur(s)}

Département des arts et lettres - Université du Québec à Chicoutimi

ISSN

0300-3523 (imprimé)

1708-2307 (numérique)

Découvrir la revue

Citer cet article

Harel, S. (2007). Répercussions violentes : Échos de l'oeuvre de Thomas Bernhard. Protée, 35(1), 17-27. https://doi.org/10.7202/015885ar
Résumé de l'article

Bien que le motif de l'écho ne soit pas abordé de manière littérale dans l'oeuvre de Thomas Bernhard, il est présent sous sa forme liminale. Le protagoniste de La Plâtrière, personnage misanthrope qui rédige un traité sur l'ouïe depuis des décennies, est sensible aux moindres répercussions de la voix humaine. Chaque son entendu par Konrad est accompagné de sa trajectoire vibratoire, ce qui crée un sentiment de désespoir profond tant la quête du silence est un exercice difficile. Dans le cadre de cet article, nous interrogerons les formes dysphoriques de l'écho : ce dernier peut représenter un monde où la répercussion violente de l'entendu provoque l'irritation. Face à ce déferlement sensoriel, dont l'écho est l'une des modalités, le sujet n'a d'autre choix que de se cloîtrer dans une maison dont il espère qu'elle l'isolera enfin du monde des vivants. De plus, la présence de tout sujet-destinataire (en l'occurrence la femme de Konrad) est la source d'une distraction sonore. Le protagoniste entend dans toute voix un écho lancinant qui retarde le mouvement de sa pensée. Il tuera sa femme, ce qui s'avère un acte criminel où le " sémanticide " apparaît de plus comme une façon d'en finir avec la présence de la voix dans le langage.
Ce document est protégé par la loi sur le droit d'auteur. L'utilisation des services d’Érudit (y compris la reproduction) est assujettie à sa politique d'utilisation que vous pouvez consulter en ligne.

https://apropos.erudit.org/fr/usagers/politique-dutilisation/ 


\section{RÉPERCU SSIO N S VIO LENTES ÉCHOS DE L'ÆEUVRE DE THOMAS BERN HARD}

SIMON HAREL

\section{L'ÉCHO: TROUBLE ET RAVISSEMENT}

Il est difficile de parler de l'écho. Trop de connotations abondent dès qu'il s'agit de cibler une aire d'intervention, un domaine d'analyse. Voilà un sujet qui semble envahi par un cortège de représentations mythologiques. L'écho nous apparait d'abord comme un bain sonore dans lequel nous vivons. Il tient lieu d'énigme, voire de surprise: entendre sa voix répétée sous la forme d'un écho, n'est-ce pas se prêter bon gré mal gré à un étrange dédoublement de la personnalité? Comme d'autres phénomènes qui modulent l'organisation de notre vie psychique, l'écho suscite à la fois un trouble et un ravissement. Le fait d'entendre sa voix, avec un léger décalage qui tient aux aléas de l'acoustique et de l'œkoumène sonore dans lequel nous sommes en quelque sorte immergés, est en soi une surprise renouvelée.

Lors de ces moments féconds, nous vivons un «stade du miroir" à rebours. Celui qui projette sa voix dans l'espoir d'en entendre l'écho souhaite s'écouter. Il donne crédit à l'unicité de la voix à cette différence près que la dualité est à l'avantplan (la répétition différée de sa propre voix), pour ensuite laisser place à l'empreinte d'une voix immémoriale: ce que les psychanalystes identifient comme l'inscription archaïque de la langue maternelle. Ainsi, l'écoute de l'écho nous fait entendre «double» (comme on dit d'une personne qu'elle voit double). Cette dématérialisation (expression étrange, j'en conviens, puisque la voix n'est jamais autre chose que cette immatérialité "en acte») qu'induit l'écho nous dépossède de toute autonomie.

Ce changement de perspective mérite d'être noté. La référence à l'écho nous fait entrevoir une énonciation paradoxale. Celui qui projette sa voix pour en faire entendre l'écho apparaît d'abord comme un "propriétaire» du discours, un énonciateur omnipotent. Autrefois la voix nous appartenait. La référence contemporaine aux lieux d'énonciation (qui habite la théorie littéraire: de la linguistique du discours à la sémiologie) reprend cette perspective d'analyse. La voix est le soubassement de l'élocution. Mais l'écho nous trompe et nous désarçonne. Cette voix que j'entends est-elle vraiment la mienne? Ainsi en est-il de l'écho: ce dernier, nous l'avons vu, peut correspondre à un trouble de grande 
ampleur. (S')entendre, comme le permet l'écho, c'est inscrire, au cour de l'énonciation, une réverbération dont nous sommes les destinataires surpris. Dans toute réflexion sur l'écho, il faut prendre au sérieux cet infinitésimal décalage qui nous fait (nous) entendre. À cette occasion, le sujet perd de sa superbe. Il n'est plus un énonciateur omnipotent (un «propriétaire» de discours), mais le destinataire (le réceptacle?) d'une voix qui vient d'ailleurs.

Dans ce contexte, le trouble ressenti à l'audition de sa propre voix possède de nombreuses ramifications. J'ai fait état un peu plus tôt de cette surprise narcissique qui consiste à s'entendre dédoublé. Ainsi l'écho nous surprend: ce n'est pas moi qui parle, c'est l'autre. Et cette altérité révélée n'est que l'expression de mon assujettissement à une voix-native dont je sais malgré tout être l'énonciateur. En ce sens, l'écho nous condamne à la répétition de l'identique. Seul l'intervalle temporel (où joue l'effet de surprise) m'assure que cette voix, si elle est mienne, me revient par des chemins de traverse dont je ne connais pas, à vrai dire, la signalétique. S'explique ainsi le mystère de l'écho: comme toute annonciation, il est la promesse d'un sens déjà-là, d'une présence dont la manifestation est explicite. Comment se fait-il alors que la perception de l'écho suscite la jubilation, ce ravissement qui consiste à maitriser la règle d'un double-entendre? Chez les enfants, comme chez les adultes d'ailleurs, l'écho est une fête. Il nous dispose au constat que nous échappons aux contraintes de la finitude humaine, en somme que nous possédons en chacun de nous un peu d'immortalité. Si nous avons évoqué à quelques reprises la figure de la voix, à propos de l'écho, c'est que cette dernière est un signifiant natif, la consécration d'une toute-puissance pulsionnelle que nous ne pouvons négliger.

Mais qu'arrive-t-il lorsque l'écho nous inquiète, qu'il ne fait pas l'objet d'une (re)connaissance heureuse? Rien ne nous dit en effet que nous devions nous émouvoir à la perception décalée de notre voix. C'est mettre au compte de notre narcissisme une responsabilité bien trop lourde que d'affirmer que nous nous reconnaissons dans tout écho, que nous y percevons l'empreinte inaltérable de notre être au monde. Comment ne pas imaginer que l'écho puisse nous inquiéter lorsqu'il est d'origine inconnue? Imaginons un instant l'habitant d'une vallée (un pâtre, un berger) qui fait entendre, par désœuvrement, le son de sa voix. L'écho lui répond, attestant sa solitude au cour d'un univers dont l'immensité est perceptible. Ainsi l'écho est une voix entendue qui vous possède, vous ramène au plus petit dénominateur commun de votre présence au monde.

Qu'en est-il de ce berger (figure dont nous avons retenu l'aspect délicieusement anachronique) s'il entend une "autre» voix lui répondre? Cette voix ne serait-elle pas l'expression d'un proche, d'un voisin, d'un visiteur, d'un intrus dont l'arrivée est (im)prévue? Cette voix serait l'écho d'une présence étrangère que notre berger a de bonnes raisons d'interroger. Dans un tel contexte, l'inquiétude est à son comble. L'écho ne correspond pas à l'actualisation jubilatoire d'une énonciation où l'identique est à l'avant-plan. Au contraire de cette perspective, le sujet ne se reconnaît plus, il ne s'entend plus. L'écho lui dit certes qu'il existe, au cœur de cette vallée, une autre voix humaine. Mais celle-ci annonce-t-elle un visiteur bienveillant ou faut-il imaginer, perspective plus troublante, l'imminence d'un cataclysme, à tout le moins d'un désordre au cœur de la communauté dont il se croyait l'unique habitant? Ces pensées anxieuses accompagnent la mise en scène acoustique d'une voix dont il n'est pas l'énonciateur.

\section{L'ORDINAIRE DE LA COMMUNICATION}

On fera valoir qu'un tel contexte est l'ordinaire de la communication humaine. Si je parle, c'est non pas pour m'entendre, mais bien plutôt pour affirmer une disponibilité à la parole d'autrui. Nous aimons affirmer cette hospitalité pérenne de la voix comme si cette dernière était d'emblée dialoguée, offerte à tout venant. Nous aimons revendiquer cette pluralité de la voix humaine, comme si un tel discours permettait de mieux accueillir une altérité que nous percevons dans le monde du langage. 
À première vue, l'expression de l'écho nous apparaît un phénomène assez anodin qui témoigne de l'emprise du narcissisme primaire. Dans toute énonciation, nous sous-estimons la présence implicite d'un destinataire qui n'est autre que nous-même. Mais le passage du temps et la maturation psychique du sujet nous enjoignent à considérer que ce « retour de l'identique» n'est pas «normal». En d'autres termes, il faudrait concevoir, comme l'enfant qui cesse de s'émouvoir à l'énonciation de ses écholalies, que le langage est tout entier tendu vers une extériorité qui est incarnée par un destinataire obligé. L'apprentissage du langage supposerait dès lors que nous abandonnions la vieille carapace humaine du repli sur soi. Dans cette description de l'activité langagière, l'écho serait un leurre, l'expression d'une estime de soi déplacée.

Revenons à notre description toute bucolique de ce berger que l'on imagine seul au cœur de sa vallée. Celui-ci entend une voix «autre» dont il perçoit presque simultanément l'énonciation et l'écho. Imaginons, toujours dans le même contexte, un éboulement en montagne, un amas de rochers qui se défait soudainement. Le berger perçoit ce fracas sonore dont il ne peut, encore une fois, certifier la source. Dans ce contexte précis, le monde de la nature fait entendre sa voix. À la suite de ces deux événements, notre berger perçoit que sa solitude est rompue. Une voix étrangère est venue troubler son activité coutumière. Un bruit d'origine inconnue lui rappelle que son monde n'est pas à l'abri de désordres en provenance du monde naturel. Si l'on voulait adopter un vocabulaire plus général (de manière à capter l'organisation sémiotique de ces deux phénomènes), nous pourrions avancer que l'écho se manifeste sous la forme d'une structure duelle, et que celle-ci suppose l'existence d'un énonciateurdestinataire capable d'en attester l'existence. Il y a chez tout sujet cette capacité de sériation cognitive qui ne relève pas de l'évidence. Si j'entends ma voix qui est réverbérée, je dois certifier qu'il s'agit bel et bien d'un écho. La chose paraîtra entendue: mais qui m'interdit de «croire» que la réverbération d'un éboulement est une annonce en provenance d'un monde «supranaturel» qui me dicte une conduite à suivre? De même, qui m'empêche d'être charmé par l'écho d'une autre voix qui m'ensorcelle et me prédispose à suivre ses diktats?

Si je me plais à faire entendre ma voix, dans l'espoir d'en recevoir l'écho, j'adopte certes un point de vue assez conventionnel. Je me fais confiant dans l'énonciation dont je suis persuadé qu'elle saura me répondre sous la forme d'une répétition. En d'autres circonstances, une telle attitude susciterait pour le moins le scepticisme. Le grand délirant n'est-il pas certain, envers et contre tous, qu'un Autre lui parle, l'informe quotidiennement de la marche du monde, voire le persécute? Celui que l'on définit comme délirant ne fait pas autre chose qu'entendre la petite voix de sa conscience sous la forme hypertrophiée du délire. Il en va autrement de la voix de notre honnête berger qu'on ne saurait accuser de déraison.

Pourtant, comme on le verra avec l'œuvre de Thomas Bernhard, l'écho peut représenter la justification d'une haine irrépressible. Nous avons tendance, sur ces questions, à faire preuve d'une grande naïveté. L'écho serait un jeu sonore qui provoque, certes, un trouble identitaire, puisque la figure du dédoublement est centrale. Néanmoins, cette pulsion vocale ne justifierait pas d'émois plus complexes. Pourtant, notre berger, s'il entend la voix d'un «autre" accompagnée de sa réverbération sous forme d'écho, peut se voir soudainement menacé: il croyait être seul dans son domaine natif, et voilà qu'une empreinte vocale d'origine inconnue s'impose absolument.

Autrefois, le monde habité (au cœur de la vallée) était simple. Puis un «étranger» apparaît, sous une modalité assez semblable à la surprise de Robinson Crusoé qui constate l'empreinte d'un pas humain sur la plage d'une île qu'il croyait déserte. Dans ce contexte, le dédoublement est certes à l'avant-scène, mais il qualifie un phénomène nouveau. Un autre tente peut-être de communiquer sa présence pour des motifs qui demeurent encore incompris. S'agit-il d'un appel à l'aide, d'un traquenard, d'un simple jeu? En 
somme, l'écho est-il ici le support d'une intention (d'un désir véritable de communication) ou l'énonciation d'une vocalise sans autre conséquence que le maniement du monde des sons. Parions que ce berger imaginaire, que nous posons comme interlocuteur de la destination signifiante qui loge en tout écho, ne saura répondre à cette question.

L'écho fait du monde une caisse de résonance où nous ne trouvons plus nos repères familiers. Si le lieu d'énonciation est l'incarnation d'une parole que le sujet exprime sans détour, alors l'écho nous renvoie au mystère d'un propos dont la volatilité est la caractéristique première. Ainsi le sujet qui se prête au jeu de l'écho sait bien qu'il en est le point de départ. La surprise, on l'a vu, est minime. Une étrangeté somme toute rassurante est à l'avant-plan. Pour quiconque s'amuse à faire entendre sa voix sous la forme de l'écho, il faut garder à l'esprit l'idée d'une castration symbolique sans grande conséquence. Car l'écho est la promesse d'une énonciation dont la scansion fait appel à la figure réconfortante du double: il est l'aveu espéré d'une annonciation.

Nous avons fait appel à cette expression dans la mesure où elle noue la forme vocale de l'écho au souhait narcissique d'une complétude. Si le sujet, dès lors qu'il parle, doit accepter l'arbitraire du signe (qui le pose comme pion dans l'univers du langage), l'écho est la promesse d'une revenance heureuse. Le fait de parler est l'indication d'une rupture, cette castration symbolique qui nous délie du corps, qui nous fait abandonner la quiétude pulsionnelle d'une proximité avec le monde tout-puissant du besoin (et de sa satisfaction) pour d'autres horizons. Ainsi, l'écho est une voix parlée, chantée, proférée (peu importent ses modalités d'énonciation), qui nous transporte dans l'éther. Si l'écho est annonciation (il nous répète ce que nous sommes), ce transport est de plus un ravissement, un voyage aérien qui rappelle le «sentiment océanique» bien décrit par Romain Rolland lors de sa correspondance avec Freud (1993).

Il n'est pas question, dans le contexte de notre interrogation, d'une immersion dans le monde du langage, d'une plongée au cour de ce monde aquatique que Sandor Ferenczi (1992) poursuivit activement. L'écho, s'il est une mer sonore, ne nous offre pas de "contenance» narcissique, comme les psychanalystes l'ont imaginé à propos de l'univers matriciel que décrit le sentiment océanique. Ainsi, l'écho nous fait vivre de manière franche ce qu'est la mobilité de la pulsion sonore dans l'espace.

Mais l'écho nous apprend aussi ce qu'est le «retour» de la pulsion, sa répétition, néanmoins différée dans le temps, qui provoque à la fois la surprise et le ravissement. Si nous avons eu recours à la notion de castration symbolique pour mieux faire comprendre la singularité de l'écho, il faut retenir que le sujet obnubilé par l'écho est défait de son identité plénière, qu'il s'entend comme un autre. Ainsi, cette situation toute provisoire, qui provoque un affect de dépersonnalisation (correspondant à notre définition de la castration symbolique), cède le pas devant le plaisir éprouvé à entendre la répétition, ce que fait l'écho.

\section{UNE VOIX IRRITÉE}

Sur ces questions, l'œuvre de Thomas Bernhard nous est d'un précieux enseignement tant elle met en scène une voix solitaire qui s'époumone à faire entendre son irritation. De façon conséquente, l'œuvre de Bernhard nous enseigne que toute voix entendue est un acte cruel, que toute altérité est violence qui prend forme dans nos lieux habités.

La Plâtrière présentait pour lui les avantages d'une geôle de travaux forcés en quelque sorte volontaires. La cour (ai-je entendu dire à Laska) est dallée de pavés de plâtre. À la Plâtrière, Konrad pouvait marcher pendant des heures sans devenir fou - avait-il dit à Wieser - alors que dans d'autres édifices aussi spacieux ou peut-être encore bien davantage, on devenait fou rien qu'après les avoir arpentés en tous sens pendant quelques minutes. Sa tête - avait dit Konrad à Wieser - était, croyait-il, précisément faite pour des bâtiments comme la Plattrière: son corps aussi. (Bernhard, 1974: 30)

Pour les besoins de l'argumentation, rappelons la trame du roman La Plâtrière : un chercheur «fou», tel qu'on les retrouve si souvent dans les œuvres de 
Bernhard (misanthrope, amoureux de musique, de solitude et de mathématiques), vit, à la manière d'un reclus, dans une plâtrière qui vient d'être rénovée à grands frais. Le chercheur, qui est à vrai dire un dilettante, voire un raté (comme cela est fréquemment observable dans l'œuvre de Bernhard), veut composer son traité d'acoustique et tue sa femme sévèrement handicapée. Au cœur de cette démarche, le protagoniste tente de faire échec à toute intrusion sonore qui pourrait empêcher la rédaction de cette œuvre. Comme c'est le cas d'autres récits de Bernhard (je pense ici au roman Le Naufragé consacré à Glenn Gould), il y a chez cet écrivain un degré zéro de l'intranquillité sonore qui n'est jamais éradiqué malgré toutes les tentatives d'isolement et de claustration que poursuivent les personnages bernhardiens.

Cela veut dire qu'un parasite vient toujours retarder, menacer la perception de cette abstraction mathématique qu'est le son «pur» du traité d'acoustique. Le musicien enfermé dans son studio d'enregistrement, l'acousticien qui veut créer les conditions optimales de réception de l'univers sonore sont à leur manière des ascètes qui glorifient leur solitude. Comme on le voit régulièrement chez Bernhard, la revendication de la culture s'oppose, de manière assez stéréotypée, au déferlement de la logorrhée communicationnelle. Les héros bernhardiens sont des hommes seuls qui profèrent leur désarroi jusqu'au moment où le suicide représente un accomplissement.

Si tel est le cas, on ne sera pas surpris que l'apparente tranquillité des lieux soit le présage d'une catastrophe. Chez Bernhard, le parasitisme sensoriel, dont une citation extraite de La Plattrière permet de comprendre les tenants et aboutissants, est à l'ordre du jour:

"Quand je ne travaille pas mon traité sur l'oüe, avait dit Konrad à l'architecte, tout est calme, la Plâtrière est entièrement plongée dans le silence, ce silence qui la caractérise». Le calme absolu régnait quand lui, Konrad, ne travaillait pas, quand il marchait de long en large, de ci, de là, qu'il réfléchissait. "Car lorsque je réfléchis, aurait-il dit, je ne travaille pas. C'està-dire, bien entendu, je travaille en réfléchissant; mais au fond, je ne commence à travailler qu'une fois mes méditations achevées; c'est alors que je commence à travailler: mais le calme est rompu; voilà que tout à coup Höller casse du bois, ou le boulanger survient, le ramoneur, l'équarrisseur, l'ouvrier scieur survient, vous survenez, Wieser survient ou Fro survient, on commence à frapper à la porte, ma femme réclame quelque chose». (Ibid.: 65)

Dans ce passage, il est clair que la rédaction du traité sur l'ouïe est un bavardage. Il en va de même de ce travail médico-musico-philosophico-mathématique, tel que le narrateur le décrit. La pensée n'arrive pas à s'extraire de son domaine natal qu'est l'espace cérébral.

Ainsi, le lieu est toujours entravé par une ambiance délétère. Ces lieux nous sont étrangers. Le conflit est à l'œuvre dans l'irritation. Le conflit est de mise dans la scène de ménage, qu'elle soit latente ou explicite.

Car naturellement, lui Konrad aurait depuis longtemps écrit son

Traité sur l'Ouie, si elle, sa femme, ne lui avait imposé des voyages à travers le monde. Il y a déjà dix ans, il aurait $p u$ l'écrire, à Londres, à Paris, à Aschaffenburg, au plus tard à Bâle, avait-il dit à Wieser. À Fro, il disait: "Elle me demande chaque jour si je porte du linge frais et je réponds oui, alors qu'en réalité, depuis une ou deux semaines, je n'ai pas changé de chemise. Elle ne remarque plus rien, ne voit plus la malpropreté et tout le reste - plus rien». (Ibid.: 88)

Le conflit est à l'œuvre dans ce silence revendiqué de l'ascète (musicien ou philosophe) qui tolère, mais avec quelles difficultés, l'irruption du bruit.

Parfois, avait dit Konrad, il entendait rouler les avalanches, il entendait les éboulis, la glace, les oiseaux, le gibier sauvage, le vent. $\grave{A}$ force de viure dans le silence, on finissait par avoir, à la Plâtrière, un sens auditif extraordinairement aigu, une ouie particulièrement fine comme lui. Tout ce qu'on entendait, tout ce qu'on n'entendait pas, aiguisait votre ouïe à la Plâtrière.

L'ouvre de Bernhard fait entendre la destruction du silence par le bruit, la destruction du bruit par le silence, l'enchevêtrement de ces polarités qui composent un magma sonore indéchiffrable. Le 
silence incarne l'idéal d'une méditation, d'un retour sur soi, mais cette quête, encore une fois, est impossible, car penser, c'est tout à la fois s'anéantir dans un silence et faire du bruit. La réflexion de Thomas Bernhard nous ramène, à certains égards, aux propos de Nietzsche, tels que cités par Pierre Sauvanet dans «L'oreille de l'architecte». Qu'est-ce, en effet, qu'un sujet sensible à l'audition, au bruit que l'on fait? Qu'est-ce qu'un espace qui tient compte de modalités d'absorption, de réfraction ou de diffraction du son, catégories mises en relief par Sauvanet?

Cet auteur fait référence, à propos de cette acoustique qui est trop souvent oubliée dans la vie quotidienne, à un aphorisme du Gai Savoir de Nietzsche, le paragraphe 280, intitulé «Architecture des contemplatifs». Je me permets de le citer in extenso:

Architecture des contemplatifs - il serait nécessaire de comprendre un jour, et ce jour estil proche, ce qui manque avant tout à nos grandes villes: des lieux de silence, spacieux et fort étendus, destinés à la méditation, pourvus de hautes et de longues galeries pour les intempéries ou le trop ardent soleil, où ne pénètrent nulle rumeur de voiture ni de crieur, et où une bienséance plus subtile interdirait même aux prêtres l'oraison à voix haute: des édifices et des jardins qui, dans leur ensemble, exprimeraient la sublimité de la réflexion et de la vie à l'écart! Les temps sont révolus où l'église possédait le monopole de la méditation, où la vita contemplativa était toujours en premier lieu vita religiosa: et tout ce que l'église a construit dans ce genre exprime cette pensée. Je ne saurais dire comment nous pourrions bien nous satisfaire de ces édifices, même désaffectés de leur destination ecclésiale: ces édifices parlent un langage beaucoup trop pathétique et contraint en tant que maison de Dieu et en tant que lieu somptueux d'un commerce avec l'audelà, pour que nous autres sans-Dieu puissions y penser nos propres pensées. Notre désir serait de nous voir nous-mêmes traduits dans la pierre et dans la plante, de nous promener audedans de nous-mêmes, lorsque nous irions de-ci, delà, dans ces galeries et dans ces jardins. (1997: 92)

Ce propos absolument décisif de Nietzsche nous renvoie à la déréliction des lieux de méditation traditionnels. L'église, ici, n'a plus aucune pertinence puisque la vie contemplative qu'elle promeut est assujettie à une règle ascétique où la religion officielle a droit de cité. En effet, l'église n'est plus la cité; elle n'a plus le monopole du silence, de la prière et de l'intériorité. Il est fascinant de voir que Nietzsche, au cœur du modernisme architectural et du tohu-bohu de l'industrialisation massive, pressent ce qui, encore aujourd'hui, est un refoulé massif de la pensée sur le monde sonore.

Nous réfléchissons beaucoup aux formes de passage d'un lieu à l'autre, à la mondialisation comme condition d'un nouvel espéranto qui ferait sa place à la diversité. Nous réfléchissons encore plus aux formes d'un métissage dont l'expression à la fois festive et joyeuse serait le témoignage d'une incarnation dans le monde. Mais nous réfléchissons peu à la fatigue que produit cet espéranto. Nous ne prenons pas en compte la dissonance que ces voix multiples produisent et nous ne voulons pas accepter le moindre instant que cette dissonance soit la source d'une pensée migraineuse où le corps va mal, où le sommeil est, tout au plus, une respiration asthmatique. Nous ne saisissons pas que le lieu, plus que l'expression bruyante d'un espace construit, est la contre-édification d'un silence nécessaire pour pouvoir penser.

\section{UNE ÉCRITURE DÉSAFFECTÉE}

Ainsi, Thomas Bernhard a tout à fait raison de cibler la désaffection des formes contemporaines de l'habiter, tant les cathédrales, les églises et les lieux de méditation sont détruits. Pour l'écrivain, ces lieux protègent non sans peine une population affolée lors de bombardements. Les récits autobiographiques de Bernhard sont les témoignages d'un environnement cruel qui anéantit tout sur son passage. Ce qui tue les hommes et les femmes, ce sont les bombes, sans oublier le bruit et le silence qui sont les expressions d'une violence insoutenable. Ce qui provoque le suicide, c'est non seulement le désespoir individuel, mais la distraction du bruit infiniment répété sous la forme d'un écho qui renvoie le sujet à l'impossibilité d'être vraiment au fait de l'autre. À bien y penser cependant, ne suis-je pas affairé à racheter Bernhard, à 
le rendre plus aimable, plus juste, peut-être même humain? N'y a-t-il pas, dans mon éloge d'une pensée de la dissonance et de l'enfermement migraineux, ce "quelque chose» qui, chez Bernhard, nous touche de façon durable, tant nous avons perdu ce lien sensible avec le monde de la voix, de l'écoute, de l'écho qui fait mal et qui résonne au cœur de notre boîte crânienne?

L'acte de chercher un lieu propice à l'écriture est au centre de l'œuvre de Bernhard. Dans cette entreprise, il faut échapper au bruit. Pourtant, la quête d'une bien relative sérénité au cœur de paysages aussi différents que la plaine, la clairière ou la forêt laisse place, soudainement, à la brutalité de l'univers domestique. Bernhard a écrit:

Ce qui me convient le mieux, c'est d'être seul. Je tiens cet état pour idéal. Ma maison est à vrai dire une prison géante. J'aime beaucoup cela. Autant que possible des murs nus. Nus et froids. C'est d'un très bon effet sur mon travail; mes livres ou les choses que j'écris sont ainsi, pareils à mon logis. (1986: 63)

Le sujet écrit en un lieu qui le protège de l'anéantissement du monde. On quitte un lieu pour se défaire de vieilles habitudes d'écriture, on achète des maisons - ainsi Bernhard propriétaire de nombreuses maisons de campagne dans son pays autrichien natal - pour s'y enfermer, puis écrire sans relâche. Tout cela, Bernhard le résume dans cette proposition centrale:

Je cherche l'origine de ma débâcle. Je purifie, j’interviens. Mais le pays natal se révèle à celui qui veut le prendre par ruse comme l'arrogance, l'ignorance transformée en dégoût. Irrité par une substance que je ne comprends pas, je fais alterner, avec une habileté que le contact des décors de théâtre a porté à une étonnante hauteur, les saisons, les hommes, les méthodes des hommes. (Ibid.: 48-49)

Retenons cette expression: «les saisons, les hommes, les méthodes des hommes». Ici, tout est dit avec une soif d'abstraction pascalienne. Le pays natal est un lieu de décadence et d'irritation. Il semble qu'il faille haïr pour habiter cet espace natal que le narrateur ne cesse, par ailleurs, de purifier, au prix d'une obstétrique scripturaire, d'une expurgation.
Au contraire de ce que nous avons écrit à propos de la représentation mythologique de l'écho, les romans de Thomas Bernhard ne tolèrent aucune intermittence sonore. Ainsi l'écho n'est pas seulement la réverbération d'un son qui se fait entendre après un certain temps. Il est, de manière générale, tout son entendu qui se répercute de manière violente au cœur de la conscience humaine. À adopter ce point de vue, on perçoit bien que l'écho devient la forme générique de toute communication. Parler, écouter de la musique, ne sont-ce pas des activités qui font résonner en chacun de nous un écho plus ou moins facilement identifiable? Toute parole qui nous est communiquée, tous les sons entendus lors de notre activité quotidienne, au cœur des villes et du monde des objets, au premier chef de notre corps, forment une fantastique caisse de résonance, sorte de caverne primitive où le monde du sens se déploie.

À partir de ce constat, on peut avancer que la parole humaine n'est pas uniquement l'objet d'une destination qui correspond à une activité instrumentale. L'écho est une stase vibratoire, un émoi qui n'est pas sans rappeler les propositions de Julia Kristeva qui font référence à l'organisation de la chora sémiotique (1977). Cela veut dire concrètement que notre corps est un site sensoriel, une balise émotionnelle qui, plutôt que d'être fichée dans l'espace, tient lieu de forme sensible.

Dans tout acte langagier, dans toute immersion au cœur de l'univers sonore, il y a mise en scène de l'écho. Nous n'avons pas la perception intime de cet état de fait. Peut-être devrions-nous ajouter que notre vigilance nous fait privilégier les pouvoirs de l'attention (et de la concentration) au détriment des charmes de l'audition. S'il est accepté que l'écho nous provienne d'un monde lointain, qu'il se répercute de montagne en montagne jusqu'au cœur de vallées où nous le percevons avec surprise, il en va autrement de l'écho de proximité, ou si l'on veut de cette chora sémiotique qui nous fait percevoir le décalage infinitésimal entre la production du son et sa rémanence. 
On n'a pas mesuré à quel point l'écho occupe pleinement chaque moment de notre vie. Il n'y a pas en effet de sonorité pure (au contraire de ce que souhaitent les personnages bernhardiens), mais un métissage de silence(s) et de sons, de pauses et d'activités où le bruit figure à l'avant-scène.

L'énonciation sonore tout comme la réception de l'écho sont affaires de contexte. Dans ce chassé-croisé du son et du silence, l'écho incarne la plupart du temps un sous-entendu que l'on perçoit à peine. Dans le mot que j'entends, qui peut être caresse sonore ou insulte, le monde des objets m'environne, me fait tout à la fois interprétant et caisse de résonance.

Notre description bucolique du berger solitaire au cœur d'une vallée qui multiplie l'écho est donc une vue de l'esprit. Certes, l'écho peut se manifester avec grandiloquence en des lieux peu habités. Mais c'est là faire jouer un dualisme excessif. L'écho, s'il semble se manifester de façon inopinée (nous avons utilisé à cet égard le vocable chargé d'annonciation), ne se contente pas de faire irruption dans un monde autrefois déserté. L'œuvre de Bernhard nous apprend que l'écho est une profération, le spectre d'une re-dite qui conduit à l'anéantissement. Les personnages bernhardiens n'habitent pas au cour d'une nature généreuse. Au contraire de notre pâtre (qui appartient à l'imaginaire romantique d'une nature accueillante), les protagonistes bernhardiens tentent de s'isoler tant bien que mal de toute sensorialité envahissante.

Au paroxysme de cet état assurément humiliant pour lui, il était donc forcé de poser souvent les mains contre les murs, contre les parois glaciales, contre les battants glacés des portes, contre les portes de fer glacées $d u$ grenier, contre les vitres, contre les bois glacés des rares meubles encore restés à la Plâtrière, et de répéter indéfiniment les yeux clos: du calme, du calme [...]. (1974: 82)

Ces personnages tentent de faire le vide, de s'abstraire de tout lieu habité dans l'espoir que surgisse enfin une idée pure, une intellection du monde qui ne s'embarrasse pas de la sensation.

Pourtant cette dernière est constamment de mise. Le protagoniste bernhardien veut s'affranchir de la détresse humaine.
La dépression résulte des mots, de rien d'autre. À Fro, il disait il y a trois ans: "Je regardais le plafond, et tout à coup, le calme qui soudain régnait dans la Plâtrière n'a plus été ce calme sinistre auquel depuis des années je m'étais habitué, c'était un calme bénéfique. Et je me disais: pas un être humain, pas un bruit, quel état idéal! Et non: pas un être humain, pas un bruit, quelle situation épouvantable! Non, elle était bénéfique».

(Ibid. : 124)

Face à ce bruit en cascade qui devient assourdissant, la solitude incarne un monde rêvé. Phénomène étrange, l'écho (la répétition à peine différée d'un bruit envahissant) s'impose sans aucune mesure. Au contraire de la musique qui incarne le bercement de cette chora sémiotique que j'ai mise en valeur, le bruit (forme de déchet sonore) est une effraction. Chez Bernhard, il faut pouvoir taire cette violence insupportable d'un bruit parasite qui vous vrille au-dedans. Au contraire d'une pensée de l'écho, qui provoque une réverbération salutaire du soi et de l'autre, le bruit-parasite s'apparente à une migraine, à l'emprisonnement sonore au cœur d'une boîte crânienne.

Faut-il pour cette raison s'étonner si les romans de Bernhard, qui octroient une large place à la musique, font preuve d'une intolérance face à l'écho? Ce dernier est un bruit répété, réverbéré à l'infini comme si le sujet habitait un cloaque sonore. Cette dernière expression n'est pas innocente. S'il existe des boîtiers d'écriture (chers à Michel Leiris), des écrins sonores qui accueillent (chez Bernhard) la plénitude du chant opératique, d'autres espaces sont de véritables maisons d'arrêt qui immobilisent le bruit dans sa course aérienne, obligent une écriture irritée. Béton et La Plattrière de Thomas Bernard décrivent cette acrimonie qui a pour objectif de détruire la source de cette impulsion sonore.

Dans un tel contexte, l'écho est la répétition d'une sonorité qui blesse les tympans fragiles, qui provoque des accès de colère inouïe. Tout se passe chez Bernhard comme si cette matrice sensorielle, dont le caractère enveloppant est manifeste, devait faire l'objet d'une mise à mort déclarée. Bien que cela ne soit pas agréable à dire, il faut avancer qu'un sémanticide voit 
le jour avec la destruction de ce bruit-parasite qu'est l'écho. Chez Bernhard, «la» femme est la source de cet affect de colère. Elle fait office de porte-voix comme si l'écho (la représentation d'un bruit dont la répétition est à peine différée) incarnait quelque principe féminin. Bien évidemment, le stéréotype est ici manifeste. Les protagonistes féminins des romans de Bernhard habitent des maisons abominables décrites comme des lieux précaires. La plâtrière, cette maison rénovée à grand frais, laisse filtrer les sons d'un environnement délétère.

À parler franc, les bruits de la scie lui étaient indifférents, à lui

Konrad, et il ne s'en était jamais soucié. Pas plus que son propre souffle, les grincements de scie ne le gênaient, ils avaient toujours existé. Jamais il ne s'était dit: "Là, tu entends une scierie, cela t'empêche de penser", puisqu'il avait toujours vécu et pensé dans le voisinage et à proximité d'une scierie. Où qu'il séjournât, il se trouvait toujours dans les parages d'une ou de plusieurs scieries.

(1974: 25)

Il est impossible de s'y isoler, encore moins d'y penser librement. La maison de Corrections est un cauchemar architectural qui conduit au suicide la sœur à qui est offert cet habitat. Que l'écho se manifeste sous la forme d'une voix de femme n'est évidemment pas anodin. Il est question de matricide tant cette voix native doit être tue.

Dans l'œuvre de Bernhard, la voix de l'autre est intolérable. Les familles sont impossibles, dans tous les sens de l'expression, elles sont insupportables. Elles provoquent l'envie de tuer. Le couple n'est pas en reste dans cette "histoire de fou » et la scène de ménage représente dans La Plâtrière une forme de duel acoustique. Dans ce contexte, la voix de l'autre suscite un effet démesuré. À défaut de répondre à sa femme dont le protagoniste reçoit la voix comme une agression, c'est un écho discordant qui occupe l'espace. À suivre ce point de vue, le tohu-bohu qui caractérise le monde sonore de La Plâtrière diffère d'un univers plus paisible.

Ainsi, Bernhard ne cesse de mettre en relief une sensibilité maladive qui perçoit chaque bruit, chaque son, comme si l'espace habité, plutôt que de restituer les sons du dehors et ceux du dedans, provoquait une déflagration sensorielle qui évite au sujet de pouvoir penser le silence.

Hier c'était l'architecte, aujourd'hui, c'est Höller. Des centaines, des milliers de petites choses m'empêchent d'écrire l'Essai. À cela s'ajoute l'otalgie de ma femme, causée sans doute par la méthode d'Urbantschitch que j'expérimente sur elle avec acharnement.

L'œuvre de Bernhard est une lutte incessante contre le bruit ambiant, l'intrusion de parasites sonores qui s'aventurent à l'intérieur de la maison qui est alors perçue comme le contenant cérébral d'une pensée informulée.

\section{UNE CEUVRE DE TÊTE}

Chez Bernhard, la tête est un organe-moteur, la matrice du penser. L'œuvre de l'écrivain insiste à loisir sur ces images qui correspondent à une angoisse de destruction. L'homme qui se suicide d'une balle dans la tête se prend à partie. Il en va de même pour celui qui se pend et se casse l'échine. Dans les deux cas, c'est la tête, tuteur de l'acte de penser, qui est visée. La pendaison est une forme de décapitation que l'on s'administre. Il s'agit en effet de couper la tête de ses racines corporelles. Complément de ce regard trouble sur la vie, la tête est l'objet d'une érogénéité du penser, comme chez d'autres grands auteurs ascétiques qui sont d'ailleurs des références pour l'écrivain.

Glenn Gould et Ludwig Wittgenstein sont des maîtres à penser, ce qui doit à peine être perçu comme un aveu ironique. L'acte de penser coïncide avec l'impossibilité de dire. Cette posture n'a rien en commun avec l'ineffable, voire l'indicible. La référence au grand-père de Bernhard est l'aveu d'une fragilité de la condition humaine. Voilà un homme qui, toute sa vie, aura souhaité être écrivain. Voilà un homme qui aura exprimé l'impossibilité de l'œuvre, son échec.

Bernhard raconte sans relâche le durcissement de l'acte de penser qui anime le travail de l'ancêtreécrivain.

Tous les jours, à trois heures du matin mon grand-père prenait un nouvel élan. Depuis de nombreuses années, Das Tal der 
sieben Höfe, manuscrit de quinze cent pages qu'il avait conçu en trois parties, lui faisait engager le combat avec la mort à trois heures $d u$ matin. Toute sa vie, affaibli par une grave maladie pulmonaire, il avait pris pour habitude d'entamer sa journée à trois heures du matin par l'occupation mortelle d'un fanatique

d'écriture et de philosophie, de s'envelopper dans la couverture de cheval et de boucler une vieille ceinture autour de sa taille, à trois heures du matin je l'entendais dans sa chambre engager le combat avec l'impossible, avec le métier dont il n'y a absolument rien à espérer: le métier d'écrivain. (1982: 85)

Il faut écrire coûte que coûte, tracer sans relâche les mots sur la feuille de papier. Au contraire d'un indicible, l'écrivain affronte la sexualité qui est à l'œuvre dans l'acte de penser. Et le suicide, acte valorisé par le grand-père, est une manière de revendiquer une attitude dévastatrice. Chez Bernhard, il existe une filiation manifeste avec l'œuvre d'un Otto Weininger (1975). Dans les deux cas, l'acte de penser est entravé par l'abjection sexuelle de l'impureté. Chez Artaud, autre référence implicite dans l'œuvre de Bernhard, l'acte de penser est l'objet d'antagonismes majeurs. Le monde pulsionnel de la sexualité provoque une débauche, une déperdition qui interdit d'être pleinement. Dans tous les cas, il faut comprendre que la voix est le siège d'une cérébralité douloureuse. Chez Bernhard, il faut savoir penser comme il faut savoir crier. Mais l'énonciation bute sur l'acte de suicide ou le constat du désespoir.
L'œuvre de Bernhard, par l'acte de fonder une demeure qui s'avère vite un lieu de cauchemar, correspond à notre propos. À cela s'ajoute le fait que l'écho est l'expression d'un rapport de force sonore. Il prend naissance dans un milieu physique, ce qui soustend l'idée que l'écho est à sa manière une forme architecturale. À ce sujet, la rédaction de La Plâtrière et de Corrections fonde une assise architecturale intangible. Le bâti architectural conserve son autonomie, quelle que soit la nature du terrain.

On ne s'étonnera pas que Corrections de Bernhard mette l'accent sur la folie d'une maison construite à partir du constat même de son impossibilité. Le narrateur ne cesse d'affirmer la cruauté de l'acte de construire, en témoigne la mort de la sœur de Roithamer dès que cette maison lui est offerte. Rester au seuil, n'est-ce pas là tout le projet de l'œuvre de Thomas Bernhard? Être dans ce lieu de passage qui suscite l'interrogation quant aux espaces que nous ne franchissons pas, n'est-ce pas encore une fois l'expression d'une limite qui rappelle cette répercussion de l'écho? Dans cette scénographie de la méchanceté qui est le cœur de l'œuvre de Bernhard, les lieux sont les formes amères d'une incarcération. Ainsi, l'écho traduit la répétition d'une voix (la sienne bien sûr, mais aussi celle des autres) qu'on ne veut pas entendre. 


\section{ÉLÉM EN TS BIBLIO G RAPHIQ U ES}

ARTAUD, A. [2004]: Euvres, éd. établie, présentée et annotée par É. Grossman, Paris, Gallimard.

Bernhard, T. [1974] : La Plâtrière, Paris, Gallimard, coll. «Du monde entier";

- [1982]: La Cave, Paris, L'Imaginaire/Gallimard;

- [1983]: Le Souffle. Une décision, Paris, Gallimard, coll. «Du monde entier";

[1984]: Un enfant, Paris, Gallimard, coll. «Du monde entier »;

_ [1985]: Béton, Paris, Gallimard;

_ [1987]: Corrections, Paris, Gallimard, coll. «Du monde entier ";

_ [1987]: Amas et autres récits, Paris, Gallimard, coll. «Du monde entier" ;

- [1990]: Extinction. Un effondrement, Paris, Gallimard, coll. «Du monde entier";

- [1991]: Le Neveu de Wittgenstein, Paris, Gallimard, coll.

"Gallimard/Folio ";

[1993]: Le Naufragé, Paris, Gallimard, coll. "Gallimard/Folio».

\section{À PRO POS DE THOMAS BERN HARD}

Bernhard, T. [1986]: Ténèbres. Textes, discours, entretiens, Paris, Maurice Nadeau.

DiERICK, A. P. [1979]: “Thomas Bernhard's Austria: Neurosis, Symbol or Expedient?”, Modern Austrian Literature, Journal of the International Arthur Schnitzler Research Association, vol. 12, nº 1, 73-93.

FrederiCO, J. [1996]: "Heimat, Death, and the Other in Thomas

Bernhard's Frost and Verstorung", Modern Austrian Literature, Journal of

the International Arthur Schnitzler Research Association, vol. 29, n³-4, 223 -

242.

GORnER, R. [1988]: "The Excitement of Boredom - Thomas

Bernhard", dans W. G. Sebald, A Radical Stage: Theater in Germany in the 1970s and 1980s, Oxford, Berg, 161-173.

KONZETT, M. [2000]: The Rhetoric of National Dissent in Thomas

Bernhard, Peter Handke, and Elfriede Jelinek, Rochester (NY), Camden

House.

KONZETT, M. (dir.) [2002]: A Companion to the Works of Thomas

Bernhard, Woodbridge (England), Boydell \& Brewer.

LONG, J. J. [2001]: "Resisting Bernhard: Women and Violence in Das

Kalkwerk, Ja, and Ausloschung”, Seminar: A Journal of Germanic Studies, Toronto, février, vol. 37, no 1, 33-52.

MARKOLIN, C. [1990]: Thomas Bernhard et son grand-père: les grands-pères sont nos maitres, Paris, P. Horay.

NAQVI, F. [2002]: "Dialectic at a Standstill: The Discourse of Victimhood in Thomas Bernhard's Heldenplatz”, German Quaterly, automne, vol. 75, no 4, 408-421.

PRICE, D. W. [2003]: "Thoughts of Destruction and Annihilation in Thomas Bernhard”, Journal of English and Germanic Philology (JEGP), Urbana (IL), avril, vol. 102, n², 188-210.

RiEMER, W. [1992]: “Thomas Bernhard's Glenn Gould”, Osterreich in Amerikanischer Sicht: Das Osterreichbild Im Amerikanischen Schulunterricht, vol. 7, 29-39.

SFORZIN, M. [2002]: L'Art de l'irritation chez Thomas Bernhard, Arras,

Presses de l'Université d'Artois, coll. «Lettres Étrangères ».

\section{SO U RCES THÉO RIQ U ES}

Altounian, J. [2000]: La Survivance: traduire le trauma collectif, Paris, Dunod, coll. «Inconscient et culture».

AnZIEU, D. [1996] : Créer-détruire, Paris, Dunod, coll. "Psychismes».

BACHELARD, G. [1943] : L'Air et les Songes. Essai sur l'imaginaire du

mouvement, Paris, Payot.

DuMOuliÉ, C. [1992] : Nietzsche et Artaud: pour une éthique de la cruauté, Paris, PUF, coll. "Philosophie d'aujourd'hui ".

FERENCZI, S. T. [1992]: Thalassa. Psychanalyse des origines de la vie sexuelle, Paris, Payot.

Flahaut, F. [1998]: La Méchanceté, Paris, Descartes et Cie.

FREUD, S. [(1929) 1971]: Le Malaise dans la civilisation, Paris, PUF, coll. "Bibliothèque de psychanalyse".

FREUD, S. et R. ROLLAND [1993]: Correspondance 1923-1936, Paris,

PUF.

KLEIN, M. [(1957) 1978]: Envie et Gratitude: et autres essais, Paris,

Gallimard, coll. "Tel».

KRISTEVA, J. [1977]: Polylogue, Paris, Seuil;

[1985]: La Révolution du langage poétique, Paris, Seuil, coll. «Points essais", n 174 .

NietzSCHE, F. W. [(1886) 1987]: Par-delà bien et mal: prélude d'une philosophie de l'avenir, Paris, Gallimard, coll. «Folio/essais».

Rosset, C. [1998]: Le Principe de cruauté, Paris, Minuit.

SAUVANET, P. [1997] : "L'oreille de l'architecte", dans C. Younès, P. Nys et M. Mangematin (dir.), L'Architecture au corps, Bruxelles, Éd. Ousia. WeIningeR, O. [1975]: Sexe et Caractère, Lausanne, L'Âge d'homme. 